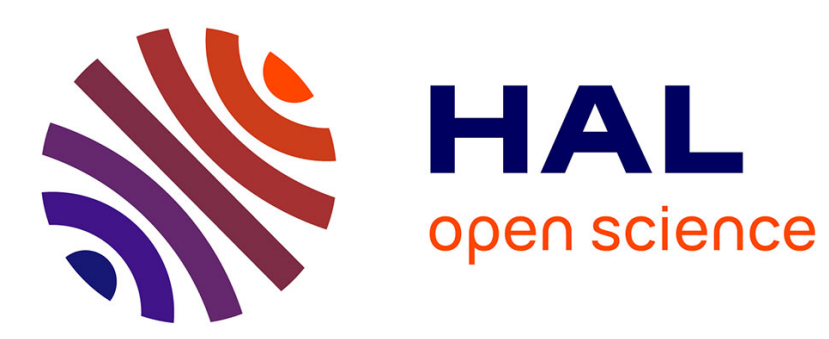

\title{
Rolling and sliding dynamics in centrifugal spreading
}

\author{
F. Rioual, E. Piron, E. Tijskens
}

\section{To cite this version:}

F. Rioual, E. Piron, E. Tijskens. Rolling and sliding dynamics in centrifugal spreading. Applied Physics Letters, 2007, 90 (2), p. 021918 - p. 021918-3. 10.1063/1.2424647 . hal-00453753

\section{HAL Id: hal-00453753 https://hal.science/hal-00453753}

Submitted on 5 Feb 2010

HAL is a multi-disciplinary open access archive for the deposit and dissemination of scientific research documents, whether they are published or not. The documents may come from teaching and research institutions in France or abroad, or from public or private research centers.
L'archive ouverte pluridisciplinaire HAL, est destinée au dépôt et à la diffusion de documents scientifiques de niveau recherche, publiés ou non, émanant des établissements d'enseignement et de recherche français ou étrangers, des laboratoires publics ou privés. 


\title{
Rolling and sliding dynamics in centrifugal spreading
}

\author{
F. Rioual, E. Piron and E. Tisjkens \\ CEMAGREF Clermont-Ferrand (Montoldre sur Allier) \\ Domaine des Palaquins, \\ 03150, Montoldre, France
}

November 20, 2006

\begin{abstract}
Centrifugal forcing is a usual method to spread a granular material. The dynamics of a particle in this system appears to change spectacularly according to the shape of the particle. We present a dynamical transition between a rolling and sliding regime and a purely sliding regime as a function of the friction coefficient and the elongation of the particle. This rolling/sliding motion is indeed observed, in-situ, for real particles. This simple effect of shape gives a physical interpretation able to explain the difference in the spreading velocity between round grains and elongated grains.
\end{abstract}


Granular flows play an important role in many of the industries, such as mining, civil engineering, pharmaceutical manufacturing as well as in agricultural processes $[1,2]$. The spreading of granulates like fertilizer and salts using a rotating disk spreader is a widespread example: what is indeed the optimal way to spread such granulates in a controlled way? This question is tough for engineers because it depends on the flowing properties of the granular medium as well as the process chosen to communicate energy to the material. In agriculture applications, the centrifugal spreader represents the main spreader chosen by farmers worldwide. In this setup, the material flows on a spinning disk and gains energy through the centrifugal force generated by the spin. Granular flows have shown to demonstrate very original properties which distinguish them from flows of liquids or gases [3]. At the root of this unique status is mostly the non-linear and dissipative nature of the interactions between the constitutive grains. Important improvements have been realized recently in the description of dense granular flows [4] as well as gravity driven dilute granular flows and a rheological description can be proposed [5]. These physical features are expected to be sensibly different for dilute flows under centrifugal forcing. From a physical point of view, the situation is indeed very original as the power force inducing the flow is a function of distance. This leads naturally to an expansion of the granular flow in such centrifugal systems contrary to dilute gravity driven flows: the distance between two grains under gravity is conserved for identical initial velocities whereas it increases exponentially for two grains under the centrifugal force. This justifies the use of the single particle approximation (SPA) where the granular flow is treated as a set of independant flowing particles and is valid for low flow rates. This letter demonstrates that in this SPA limit some individual properties of the particle as its shape have a crucial effect on the outcome of the spreading.

The model system considered here consists of a flat disk rotating at a velocity $\Omega$ with two straight vertical vanes through the disk center so that the trajectory of the particle of velocity $V$ on the disk lies in the horizontal plane. As the contact force between the particle and the vane is much larger than its weight (gravity $g$ ) (i.e., typically, $\frac{\Omega V}{g} \geq 10$ ), friction on the disk will be neglected). We assume the trajectory to be in the horizontal plane such that we can model it in two dimensions. The disk is rotating at a constant velocity $\Omega$. The outline of the nonspherical particle in contact with the vane is represented by an ellipse of small axis $a$ and big axis $b$. Its moment of inertia per unit mass with respect to its gravity center is $I$ where $I=\frac{1}{2}\left[a^{2}+b^{2}\right]$ in $2 D$ and the volume of the particle is fixed. The contact between the particle and the boundary is treated as a point in the framework of rigid body dynamics (contact point noted I).

The forces acting on the particle are the coulombian friction force $(T=$ $\mu N)$ with the boundary and the inertial force (centrifugal and coriolis). In the general case, the particle can roll as well as slide and the trajectory of 
its center of gravity $\mathrm{G}\left(x_{G}, y_{G}\right)$ is solution of the three following equations of Newtonian mechanics:

$$
\begin{aligned}
-\frac{T}{m}+x_{G} \Omega^{2}+2 \Omega \frac{d y_{G}}{d t} & =\frac{d^{2} x_{G}}{d t^{2}} ; \\
\frac{N}{m}+y_{G} \Omega^{2}-2 \Omega \frac{d x_{G}}{d t} & =\frac{d^{2} y_{G}}{d t^{2}} ; \\
y_{G} T+\left(x_{G}-x_{I}\right) N & =I \frac{d^{2} \theta}{d t^{2}} ;
\end{aligned}
$$

We suppose the contact to be punctual. The contact point $I$ belongs to the outline of the elliptic particle and the plane of the disk which induces two aditional equations:

$$
\begin{array}{r}
y_{G}=+b \sqrt{1+\frac{a^{2}-b^{2}}{b^{2}} \sin ^{2} \theta} ; \\
x_{G}-x_{I}=\frac{\left[\left(\frac{1}{a^{2}}-\frac{1}{b^{2}}\right) \sin 2 \theta \Psi(\theta, a, b)\right]}{2\left[\left(\frac{\cos \theta}{a}\right)^{2}+\left(\frac{\sin \theta}{b}\right)^{2}\right]} ;
\end{array}
$$

We solved numerically the dynamical system for the 6 coupled equations with a Runge-Kutta method of rank 4 (unit of space: $r$; unit of time $\Omega^{-1}$; linear velocity $r \Omega$; unit of angular velocity $\Omega$ ). (Initial conditions chosen for the particle are $\left.\theta=0 ; \frac{d \theta}{d t}=0 ; \frac{d^{2} \theta}{d t^{2}}=0 ; x_{G}=x_{I}=12 r ; \frac{d x_{G}}{d t}=1 e-5\right)$. We studied the system with respect to the two parameters describing the particle: the elongation $s=\frac{a}{b}$ and the friction coefficient $\mu$.

At a constant friction coefficient, increasing elongation $s$ from 1 , we observe a regime $(R+S)$ in which the particle rolls and slides at the same time. For a value of the elongation $s$ higher than a critical value $s^{\star}$, we observe a transition towards a regime $(S)$ in which the particle is not able to roll (the spinning velocity goes to zero) and takes a motion of a pure sliding. The linear velocity of ejection $V_{l}=\frac{d x_{G}}{d t}$ is subjected at the same time to a decrease of the order of $20 \%$ at this transition at the end of the disk. The particle in a pure sliding regime $(S)$ takes a weak constant inclination $\left(\theta^{e q}=f(\mu, s)\right)$. Indeed, the sliding regime $(S)$ appears only if equation (4) vanishes. As $0<\mu<1$ and $s>1$, a pure sliding regime $(S)$ appears if: $s>s^{\star}$ with $s^{\star}=\frac{1}{\sqrt{1+2 \mu^{2}-2 \mu \sqrt{1+\mu}}}$. The origin of the transition appears on equation (4): the dynamics in the regime $(R+S)$ or $(S)$ results in fact in a competition between the moment of the friction force on the particle ( first term of equation (4)) and the moment of the normal force (second term of equation (4)). Experimental measurements have been realized for two types of grains a- spherical, well calibrated, beads ( $B B$ type) $(d=5,93 \mathrm{~mm} . \pm 0,02)$ b- a sample of elongated $K C l$ fertilizer particles $(d=3,21 \mathrm{~mm} . \pm 1,13)$. We checked also a weak difference of the friction coefficients for the two samples: $\mu_{B B}=0,47$ and $\mu_{K C l}=0,52$ at an imposed normal force on a 
particle equal to the coriolis force at the edge of the vane $(N=0,4 N ., 1 \mathrm{~m} / \mathrm{s})$ producing a difference less than $4 \%$ according to the model. Experiments (figure 3) were performed on a rotating disk $(L=20 \mathrm{~cm}$., $\Omega=400 \mathrm{rpm}$.) and required a camera working at a frame rate of $250 \mathrm{~Hz}$. We release a particle, at low energy, at a constant distance from the centre of the disk $\left(x_{G 0}=3,6 \mathrm{~cm}.\right)$. By image analysis, we did a statistical analysis on thirty samples and obtained the velocities $V_{B B}=8,74 \mathrm{~m} / \mathrm{s} ; \Delta V_{B B}=0,81 \mathrm{~m} / \mathrm{s}$ and $V_{K C l}=7,54 \mathrm{~m} / \mathrm{s} ; \Delta V_{K C l}=1,85 \mathrm{~m} / \mathrm{s}$ corresponding to a relative variation of the velocity between $B B$ beads and $K C l$ beads equal to $16 \%$; a result in agreement with our model based on the shape effect. Stroboscopic observations of the motion of a partly coloured particle along the vane shows indeed the absence of rotation of the particle for $\mathrm{KCl}$ beads contrary to $\mathrm{BB}$ spherical beads (see figure()) confirming the crucial role of the shape of the particle in the dynamics.

Some crucial features of the centrifugal spreading are directly connected to some physical caracteristics of the particle such as its main elongation and its friction properties along the vane. These parameters are indeed controlling the dynamics of the particle between a rolling/sliding regime $(R+S)$ and a purely sliding regime $(S)$ for the particle along the vane.

Acknowledgements This work has benefitted from interesting discussions with the members of the T.S.C.F. group of Cemagref. P. Héritier is acknowledged for its help in performing the measurements of the friction coefficient with the viscosimeter. E. Guyon and J.C. Baudez are acknowledged for a critical reading of the document and $H$. Cherasse for technical assistance. 


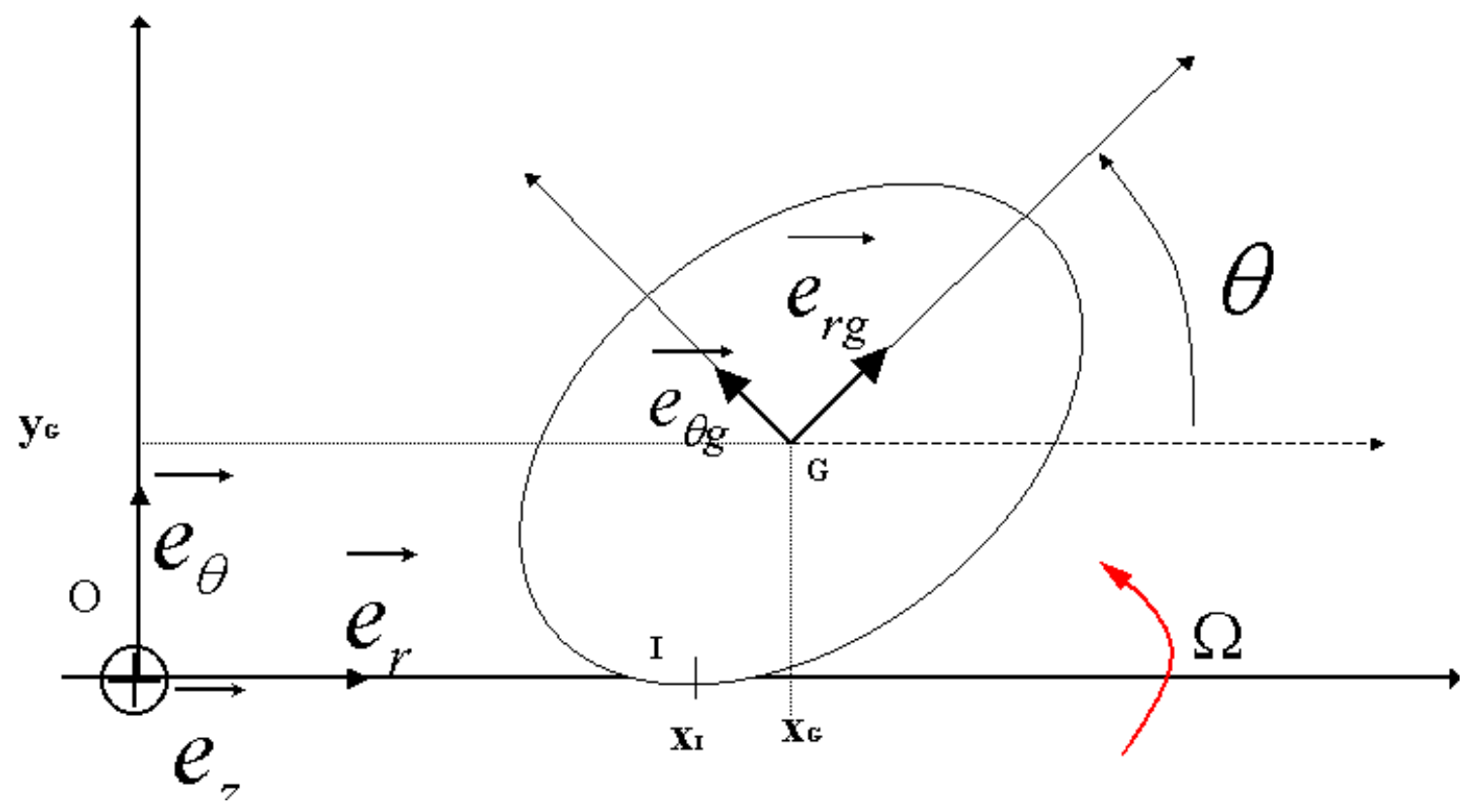

Figure 1: 
Applied Physics Letters, 2007 vol. 90, $\mathrm{n}^{\circ}$ 2, 021918 doi : 10.1063/1.2424647

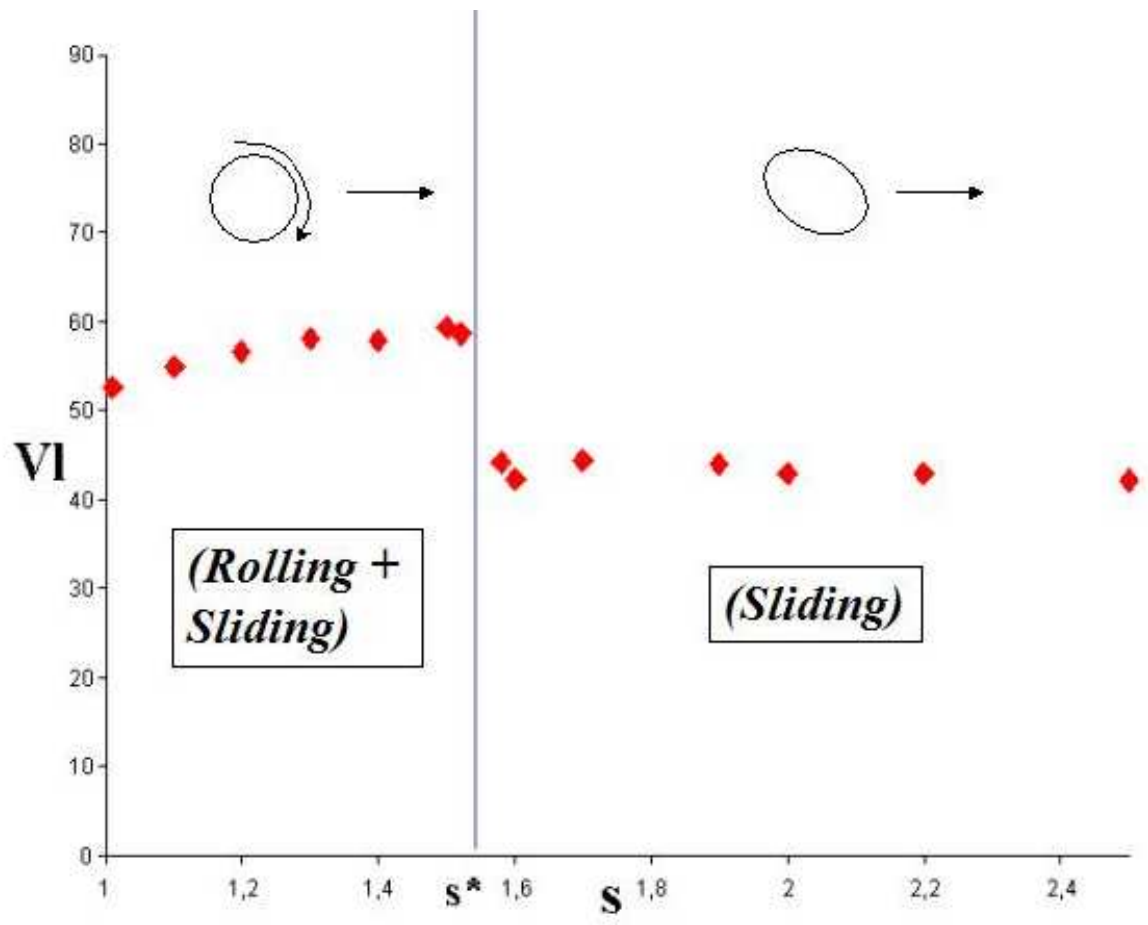

Figure 2: 


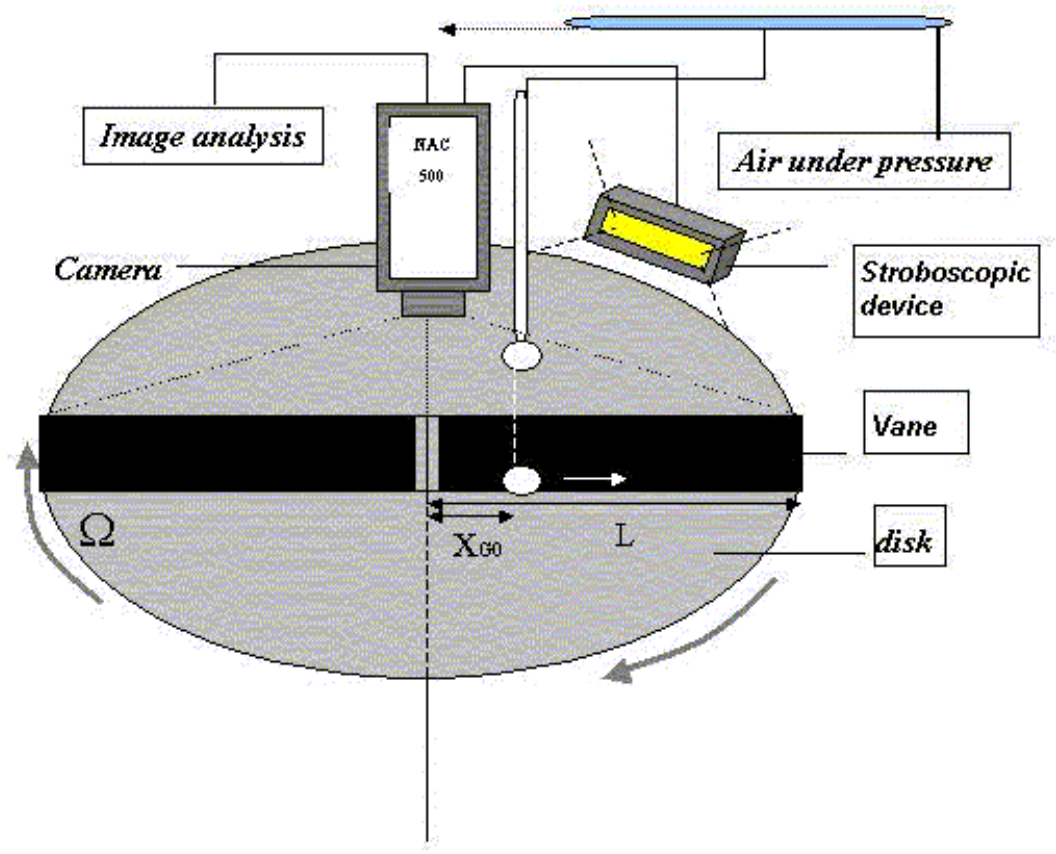

Figure 3: 
Applied Physics Letters, 2007 vol. 90, n² 2, 021918

doi : 10.1063/1.2424647

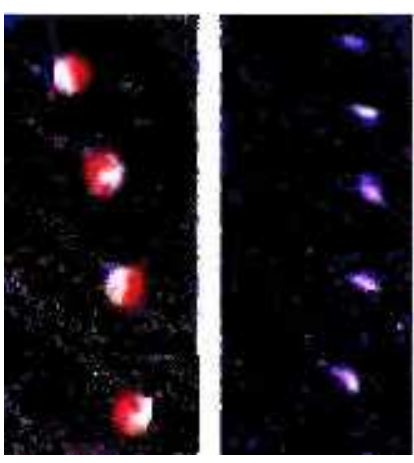

Figure 4: 
Figure 1: Sketch of the particle in the rotating frame

Figure 2: Linear velocity of ejection $\mathrm{Vl}$ (for $\mu=0.4$ )

Figure 3: Experimental setup

Figure 4: A rolling and sliding $B B$ bead (left); a sliding $K C l$ bead (right) 


\section{References}

[1] E.Guyon and J. Paul Troadec, Paris: eds. Odile Jacob (1994)

[2] J. Duran, New York: Springer Verlag (2000)

[3] H.M. Jaeger, S.R. Nagel, R.P. Behringer, Reviews of Modern Physics, 68:1259-1273 (1996)

[4] O.Pouliquen, C.Cassar, P.Jop, Y. Forterre and M. Nicolas, J. Stat. Mech. P07020 (2006)

[5] I. Goldhirsh, Ann. Rev. of Fluid Mech. Vol. 35: 267-293 (2003)

[6] Tijskens, E., Ramon, H., De Baerdemaeker, Journal of Sound and Vibration; 266: 493-514 (2003) 